present condition. The appetite and digestion are good and the weight normal. The pulse is 110 and his temperature 100 . Upon examination of the chest the area over which the heart's impulse can be seen is found to be enlarged, being at least four inches in diameter; but the strongest impulse, indicating the position of the apex of the heart, is nearly in its normal position, though the extreme line of cardiac dulness as marked out by the consulting staff extends one and a half inches to the left and about as far below the normal position. Upon palpation I find the greatest force of the impulse half an inch to the left of the maxillary line. Upon auscultation I heard a distinct systolic murmur in the second intercostal space of the right side close to the sternum, and an impurity of the first sound of the heart is heard over a greater portion of the aortic area. This is due to some obstruction in the aorta or at the aortic valves, which condition is probably the main factor in the enlargement of the heart. Further examination shows that the left side of the chest is three-fourths of an inch smaller than the right, but the expansion is the same upon both sides. Dulness is found over the upper part of the left lung, extending down to about the fourth rib in front and to the fifth rib behind. In this region the expiratory murmur can scarcely be heard, but there are no râles. The vocal fremitus appears to be a little exaggerated over the whole of the left side. Over the lower third of the left lung there is also some dulness with a very feeble respiratory murmur, but over the middle portion of this lung the respiratory sounds are much more distinct. The sounds at the lower part of the left lung are about onethird as intense as upon the right side. Over the upper portion of the right lung the respiratory murmur is diminished in intensity, but the quality is normal. Over other parts of this lung the sounds are natural or slightly intensified. It has been suggested that the cardiac symptoms and signs in this case are due to simple hypertrophy, but with an uncomplicated hypertrophy we ought not to find a pulse of 116 and the patient ought not to be annoyed by palpitation; beside, the impulse would be considerably stronger than we find it in this case. The heart is evidently both hypertrophied and dilated and, as already stated, this condition is doubtless largely due to the obstruction in the aorta, but it is probable that the obstructed pulmonary circulation is partly responsible.

While there is no doubt about the condition of this man's heart and about the consolidation of his lung, there is still much reason for speculation as to the cause of his trouble. The patient cells us that the disease came on at first with a sharp pain in the side, and says that this pain lasted for a few days and then became less and less severe, but that it has continued to some extent ever since. It would appear probable that he suffered first from an acute pleurisy, but that it became chronic and that the effusion was not absorbed for several weeks or possibly months. During this time the inflammation of the pleura gradually extended to the lung itself, the intervesicular tissues being involved, and finally the new connective tissue contracted and obliterated most of the air cells in the apex and a considerable number of those in the base of the left lung. This is not an uncommon condition after pleurisy. The temperature in this case may be due either to the condition of the lung, or to endocarditis or myocarditis. The continuous pain from which the patient suffers in the region of the heart would suggest that inflammation may be still going on in that organ. The cough and expectoration, however, would suggest that the rapid pulse and increased temperature are the result of changes in the lung.

The sputum has not been examined, therefore we can not tell whether tuberculosis has set in, though as I have already told you, it is almost sure to occur sooner or later when chronic inflammation of the lung follows pleurisy. In the absence of a positive diagnosis we will prescribe for this patient 5 minims of the tincture of digitalis to strengthen the heart action, 30 minims of the tincture of hyoscyamus to lessen the cough, and 5 grains of the iodid of ammonium not only to lessen the cough but to diminish the cardiac pain. These will be given in syrup of glycyrrhiza three or four times a day. If a microscopic examination should reveal to us the presence of tubercle bacilli, the proper treatment for tuberculosis would be given in additicn to that for the heart. It is so unusual for pulmonary tuberculosis to occur in connection with chronic cardiac disease that it is sometimes assumed that the two are antagonistic If this man were 45 years of age, we might well inquire whether the pain and rough sounds over the aorta were not due to slight dilatation or to an aneurysm of that vessel, but as he is only 21 , I think it more reasonable to attribute them to changes in the heart itself. The prognosis as to recovery is necessarily unfavorable, but unless some accident occurs, we believe that this patient may hope to live at least three or four years.

\section{REPORT OF A CASE OF GUNSHOT WOUND} OF THE CHEST.

BY J. F. JENKINS, M.D.

THCUMSEH, MICH.

In the evening between the hours of 8 and 9 o'clock of April 26, 1896, a messenger came to my office from Dr. O. N. Rice of Tipton, some six miles distant, requesting me to come to his assistance; that there were two persons in that hamlet who had recently received gunshot wounds.

Eugene C., age 50 years, weight about 200 pounds, was shot in the chest while his assailant stood some fifteen feet from him; after shooting C., the young man fired a bullet through his own head, dying within an hour afterward.

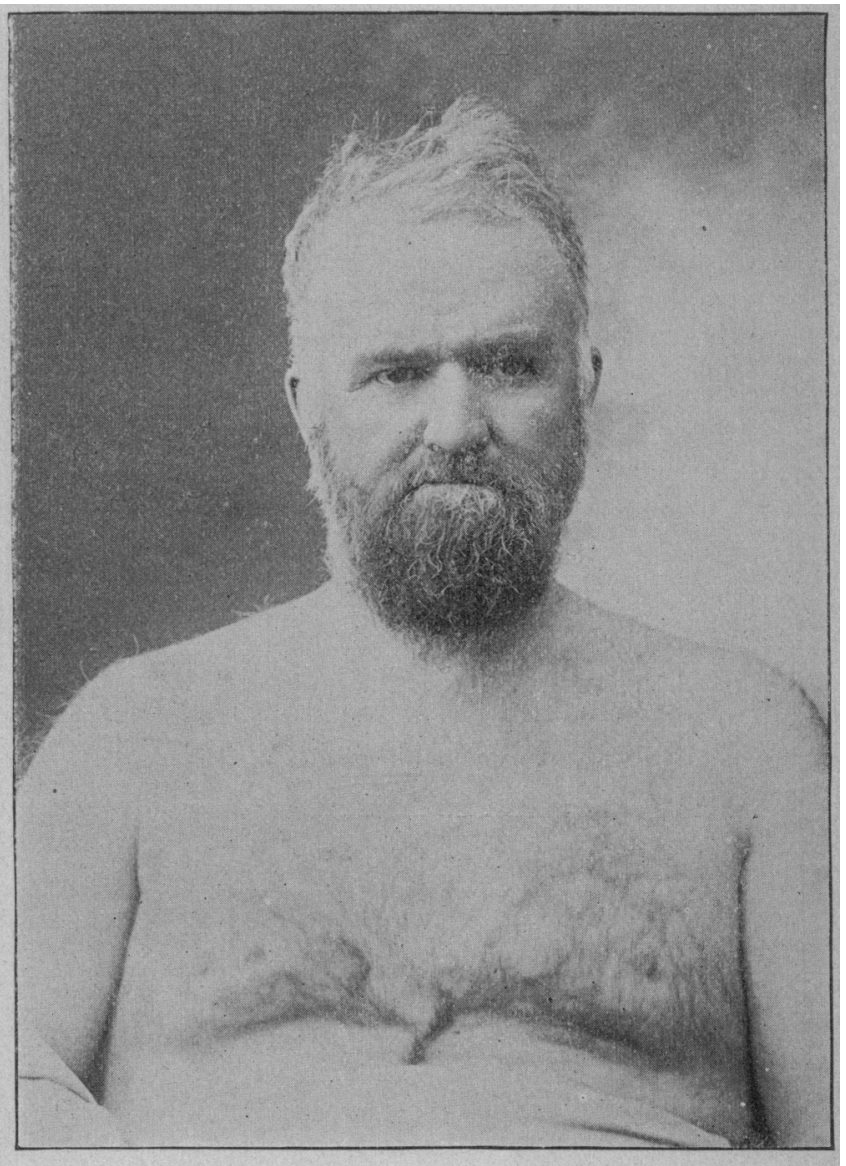

The revolver was a 32-caliber, and the bullet struck C., as the reader will observe from the photograph about two inches to the left of the right nipple passing through the lung, and although the missile has not been extracted, the present indications are that it is buried in the tissues on the anterior surface of the right scapula near its inferior angle. An hour after receiving the wound, the patient was breathing rapidly and was suffering from shock. A careful search was then made for the bullet by pinching up the skin and tissues beneath, but with negative results. The wound of entrance was neither probed nor examined by the finger, and notwithstanding considerable hemorrhage occurred from the wounding of the intercostal artery, yet the bleeding subsided spontaneously. The cutaneous surface about the wound was shaved and thoroughly cleansed, the wound dressed antiseptically, and the 
patient placed in a semi-recumbent position; hypodermics of morphin were given occasionally, sufficient to relieve pain. A moderate hemoptysis took place which continued more or less until the fourth day. On the following day upon an examination of the chest, there was found a circumscribed pleuritis and traumatic inflammation of the lung extending downward from the second intercostal space on the right side.

Twenty-four hours after receiving the wound, the temperature of the patient was 100 degrees, respiration 40 per minute, and pulse 102. Absolute quiet and an expectant course of treatment was adopted; antiseptic dressing was applied to the wound, which healed without suppuration. On the eighth day the temperature was 99.4, pulse 84 , and respiration 24 per minute.

In the following night the patient complained of severe pain along the posterior portion of the right leg below the knee, and when examined was found swollen, the skin was pale and cool; there was tenderness along the course of the external saphenous vein, which felt like a whip cord. The phlebitis of the external saphenous was doubtless caused by a septic embolism. During the succeeding ten days the temperature varied from 99 to 102 degrees, respiration from 20 to 26 , pulse from 84 to 110 per minute. The limb was elevated, and warm applications were made to the extremity; frequently the limb was immersed in hot water from ten to fifteen minutes at at time. A supporting course of treatment was followed, and sufficient anodynes to control pain.

Four weeks after the shooting, the respiratory murmur could be distinctly heard over the greater part of the lung, excepting along the course of the bullet, the phlebitis had subsided, and the patient discharged by Dr. O. N. Rice, who skillfully conducted the case, and who has kindly given me his history after my second visit.

A CASE OF FLAT RACHITIC PELVIS; PRO. LAPSE OF THE CORD WITH THE HEAD; VERSION AND EXTRACTION. Read at the Meeting of the Obstetric Staff of the Chicago Health
Department, Nov. 21,1896 .

BY JOS. B. DE LEE, M.D.

Lecturer on Obstetrics, Northwestern University Medical School, Obstetrician to Mercy Hospital, to the Chicago Lying-in Hospital, etc. CHICAGO.

Mrs. H., iv-para; two forceps deliveries: one craniotomy; one spontaneous delivery, child living.

Present labor at term, began Nov. 9, 1896, at 9 P.M. Dr. A. E. Coy was called before midnight and found the cervix admiting two fingers, cephalic presentation and a large loop of pulsating cord in the vagina. Pains were strong and soon dilated the cervix.

At 2 A.M. Dr. Coy kindly invited me to see the case.

Patient is a large, strong, apparently well built Irish woman.

Spines, 281/2 ; crests, 31 ; trochanters, 34, / ; Baudelocque, 19 ; conj. diag., 11 : conj. vera, estimated at 9 to $91 / 4 \mathrm{~cm}$.

Position, O. I. A. ; heart tones, 140 per minute, strong and regular; head freely movable above the inlet.

Internally - perineum torn deeply at some previous labor, cervix thick, os admits the hand easily, old scars in the vagina, head movable above the inlet, sagittal suture transverse, small fontanelle at left, large to right and lower than the other. Pelvis roomy below and at the sides, contracted in the conjugate diameter.

There were two methods of treatment open to us, first, reposition of the cord and bringing it over one leg; second, version and following this, extraction.

Usually it lies with the choice of the operator or his individual skill, which one of these procedures he adopts, but in this case the presence of a contracted pelvis, as evidenced the pelvic measurements and the history of the case, decided in favor of the version.

The patient was put on the table, anesthetized and the parts sterilized. The anterior foot was now brought down, but the version could not be com. pleted as the head could not be dislodged from the inlet. A maneuver known as that of Justine Sigmundine was now tried. A stout sling was put on the foot and while pulling on this with one hand the head was pushed up from below with the other. Version completed, after which the heart tones were rapid but otherwise good. We waited fifteen minutes and undertook the extraction, for which the patient was allowed to awaken from the chloroform. It required considerable traction aided by pressure from above to deliver the trunk, and the armis were thrown up alongside the head so that great difficulty was met in delivering them.

The head was arrested at the inlet, Smellie-Veit maneuver and strong pressure from above brought it down to the vulva, where it was found that the cervix had contracted tightly around the head. Chloroform was again given and the cervix slowly worked upward by the fingers. By separating the walls of the vagina and vulva and wiping the passage clear, air was allowed to the child's mouth.

After delivery the trachea was cleared with the tracheal catheter and the slightly asphyxiated child revived by the usual methods. It was a female, weight $7 \frac{1}{2}$ pounds, length $50 \mathrm{~cm}$. The puerperium was entirely normal.

Among the many interesting points this case presents, a few may be touched on. The maneuver invented by Justine Sigmundine, "Royal Midwife to the Brandenburg Court," is of signal use in cases of difficult version. Where the head is held tightly against the inlet or in the lower uterine segment, enough traction may be put on the leg as to break it without dislodging the head, even when the traction is aided by upward pressure against the head from the outside. One may also bring down the other leg and try the turning on the two. The little maneuver just mentioned is simpler and the following extraction is rendered easier if the other limb be allowed to remain in place with the breech and thus dilate the cervix more (that is, if the extraction be made on one leg). In the hardest cases it may be necessary to put slings on the two legs and push up the head with the hand in order to complete the version.

Where, in the interest of the child, an extraction by the breech is undertaken before the cervix is com. pletely dilated, it may happen that the cervix, irritated by the passage of the child through it or by many and hasty manipulations with the hands, closes down on the head of the child, button-holing or imprisoning it in the uterus. Under these circumstances the child is lost, if it is not given air, in a very few minutes. At the risk of tearing the cervix and of grave post-partum hemorrhage one may forcibly pull the head out. In these cases, traction brings the head to the vulvar outlet and the cervix may be seen. Under chloroform the fingers may slowly work the constricting edge over the face, and the mouth now being free, it is cleansed and air allowed to it by separating the labia and vagina with the fingers. In this way the child may breathe while the cervix is given time to relax and slip back off the head. Carl 